\title{
Defectos congénitos cráneo-encefálicos: variedades y respuesta a la fortificación de la harina con ácido fólico
}

\author{
Edgardo Corral S $\mathbf{S}^{1}$, Rodrigo Moreno $\mathrm{S}^{2}, \mathrm{G}$ uillermo Pérez $\mathrm{G}^{3}$, \\ María Elena 0 jeda $B^{1,4, a}$, Hernán Valenzuela $G^{1}$, \\ Mauricio Reascos $M^{6}$, Waldo Sepúlveda $L^{5}$. \\ Effect of flour folic acid fortification \\ on the incidence of cranio \\ encephalic congenital defects
}

Background: In Chile, flour is fortified with folic acid since 2000, and the incidence of neural tube defects has decreased. Cranio-encephalic congenital defects (CECD) have a wider clinical scope, includind classical neural tube defects (NTD) such as anencephalia and acrania, but also incorporating other defects. Therefore, the effects of folic acid fortification on the incidence of CECD could be different. Aim: To study the effect of folic acid fortification on the incidence of CECD. Material and methods: All prenatal diagnoses of CECD and cases registered locally in the Latin American Collaborative Study of Congenital Malformations (ECLAMC) were reviewed. The obtained data were crossed with all death certificates of newborns or stillborns of more than $500 \mathrm{~g}$ in the Sixth Region, in Central Chile. All cases were classified again in four subtypes, according to their phenotype. The incidences of all subtypes prior (1996-2000) and after folic acid supplementation (2001-2004), were compared. Results: Between 1996 and 2004, there were 64 cases of CEDC among 109,176 infants born alive. The global rate was 8.86 per 10,000 born alive in the period prior to fortification and 3.03 per 10,000 born alive after fortification. Comparing both periods, there was a 66\% reduction in all CECD and a 77 and $100 \%$ reduction in subtypes A and B of CECD, respectively ( $p \triangleleft 0.05$ ). No significant reductions in type C and D CECD, were observed. Conclusions: After the start of flour folic acid fortification, there was a global reduction in the incidence of CECD. However, only types A and B CECD, supposedly more responsive to folic acid, were reduced (Rev Méd Chile 2006; 134: 1129-34).

(Key words: Dietary supplements; Flour; Folic acid; Nervous system malformations)

\footnotetext{
Recibido el 8 de abril, 2005. Aceptado el 28 de marzo, 2006.

${ }^{1}$ Servicio de Obstetricia y Ginecología, Unidad de Ecografía, Hospital Regional, Rancagua;

${ }^{2}$ Unidad de Genética, Hospital Regional, Rancagua, Universidad de Chile; ${ }^{3}$ Servicio de Anatomía Patológica, Hospital Regional, Rancagua; ${ }^{4}$ ECLAMC (Estudio Colaborativo Latino Americano de Malformaciones Congénitas); ${ }^{5}$ Unidad de Medicina Materno-Fetal, Departamento de Obstetricia y Ginecología, Clínica Las Condes, Santiago. ${ }^{6}$ Programa de Formación como Especialista de Obstetricia y Ginecología. Universidad de Santiago de Chile. aMatrona
}

Correspondencia a: Dr. Edgardo Corral Sereño. Unidad de Ecografía, Servicio de Obstetricia y Ginecología, Hospital Regional, Rancagua. Fono Fax. (072-234803). E mail: galocorral@entelchile.net 
$\mathrm{E}^{1}$ desarrollo comunitario produce un cambio epidemiológico en la morbimortalidad infantil y perinatal, disminuyendo la incidencia de las enfermedades infecciosas 0 la malnutrición y emergiendo las alteraciones genéticas, metabólicas y malformativas. Actualmente, Chile presenta cifras de mortalidad infantil y perinatal muy cercanas a las que muestran los países desarrollados. En el año 2000, la tasa de mortalidad infantil fue de 8,9 x $1.000 \mathrm{NV}$, siendo las malformaciones congénitas responsables de $34 \%$ de la mortalidad infantil ${ }^{1}$.

Los defectos del tubo neural (DTN) son una de las anomalías congénitas más frecuentes en nuestro medio y son conocidas clásicamente como: anencefalia, cefalocele y espina bífida. Típicamente, se describen como anomalías de origen multifactorial (poligénico-ambiental), aunque en algunos casos existe asociación con ciertos síndromes genéticos (Meckel-Gruber, Jarcho-Levine, Wardenburg) o con anomalías cromosómicas (trisomía 18). En nuestro medio se ha comunicado una prevalencia de anomalía cromosómica en DTN de $2 \%$ para anencefalia, $9 \%$ para espina bífida y de $14 \%$ para cefalocele ${ }^{2}$.

De los factores ambientales estudiados, el aporte periconcepcional de ácido fólico (AF), ha sido el único que ha demostrado tener impacto como medida de prevención primaria para disminuir la incidencia de los DTN. En Chile, por decreto ministerial se fortifica la harina (220 ug de AF por cada $100 \mathrm{~g}$ de harina) desde enero del año $2000^{3}$.

Las publicaciones del ECLAMC (Estudio Colaborativo Latinoamericano de Malformaciones Congénitas), para el periodo 1982-1999 (periodo prefortificación), muestran que los DTN en Chile tenían una tasa de 19,9 x 10.000 RN. Para el Hospital Regional de Rancagua se observaron cifras aún mayores, 31,7 x $10.000 \mathrm{RN}$ y tanto la prevalencia de anencefalia como de espina bífida, eran estadísticamente superiores respecto del resto de hospitales del ECLAMC. La anencefalia en particular, presentó en el mismo período, una prevalencia al nacer de 12,2 x $10.000 \mathrm{RN}$ y era prácticamente el doble de la del ECLAMC, donde esta anomalía se ha mantenido muy estable los últimos años $(6,9 \times 10.000 \mathrm{RN})^{4,5}$. Un estudio transversal de 9 maternidades de Santiago, con aproximadamente 60.000 nacimientos, mostró durante el año 1999, una incidencia de DTN (solo o asociado a otras malformaciones) de 1,56 x 1.000 $\mathrm{RN}$ y de $0,62 \times 1.000 \mathrm{RN}$ para la anencefalia sola ${ }^{6}$.
Sin embargo, los estudios para comparar frecuencias antes y después de la fortificación de harina con ácido fólico, son aún escasos. Un trabajo del ECLAMC, que incluye la anencefalia (sola o asociada), muestra que las tasas no estaban disminuyendo en Chile antes de iniciar la fortificación, y que la disminución posfortificación fue aproximadamente de 51\% para espina bífida y de $46 \%$ para anencefalia. Para el Hospital de Rancagua se publicaron tasas de anencefalia de 11,53 x $10.000 \mathrm{RN}$ en el periodo prefortificación y de 2,95 x $10.000 \mathrm{RN}$ para el periodo posfortificación ${ }^{7}$. Un estudio nacional preliminar mostró en la Región Metropolitana, una disminución global de 38,6\% para todos los DTN entre ambos periodos. La tasa de anencefalia fue de 5,5 y 3,7 por $10.000 \mathrm{RN}$ para el periodo pre $\mathrm{y}$ posfortificación, respectivamente. Estudios locales han mostrado incluso una disminución mayor, luego de aplicadas las medidas de fortificación ${ }^{8,9}$.

La identificación de mutaciones génicas de algunas enzimas del metabolismo de los folatos-homocisteína, permiten suponer que algunos defectos del tubo neural podrían ser la expresión de un error innato del metabolismo de estas vitaminas, que actúan en un periodo muy crítico de la neurulación primaria. Una de estas mutaciones da como resultado una enzima defectuosa svariante termolábil», que exhibe menos de $50 \%$ de la actividad de la forma alélica normal ${ }^{10,11}$. En nuestro medio también se ha informado de una alta frecuencia del polimorfismo genético del gen de la enzima metilen tetrahidrofolato reductasa (MTHFR), una de las enzimas más importantes vinculadas con el metabolismo del AF y por lo tanto con las síntesis de purinas y $\mathrm{ADN}^{12}$. Así, una condición genética predisponente, asociada a una ingesta inadecuada de folatos, podría ser responsable de a lo menos $50 \%$ de los DTN.

Históricamente, los DTN han sido clasificados en forma muy general, considerándolos a todos como del mismo tipo. Esta clasificación genérica y escueta en sus descripciones considera erróneamente, por ejemplo, a todas las acraneas y anencefalias como equivalentes. Ambas malformaciones constituyen entidades fenotípicamente diferentes y responden a estructuras embriológicamente separadas, aunque ambas están relacionadas con la neurulación primaria. La acranea es una anomalía de las crestas neurales y la anencefalia, (que habitualmente observamos), constituye generalmente una «secuencia», donde el defecto primario 
es la ausencia del cráneo y donde secundariamente se destruye el encéfalo. Existen otras condiciones en las cuales el cráneo se encuentra inicialmente ausente, pero el encéfalo adherido por bridas a las paredes uterinas, se encuentra intacto. Estas condiciones permiten suponer que no todos estos defectos tendrían que ser corregidos en la misma proporción con el mayor consumo de AF.

Los defectos congénitos cráneo-encefálicos (DCCE) constituyen una malformación fácil de reconocer en un examen pre y posnatal, y por lo tanto, pueden ser usados como un ejemplo clínico confiable para conocer la frecuencia real de una malformación y establecer las diferencias antes y después de una medida.

Los propósitos de este trabajo son: 1) conocer la incidencia regional de todos los defectos congénitos del polo cráneo-encefálico, 2) revisar la clasificación habitual de la acranea/anencefalia y proponer una reclasificación de estos defectos, 3) calcular y comparar las tasas globales y por subtipo para los periodos pre y posfortificación, 4) evaluar el impacto global y específico de la medida en cada subtipo, luego de aplicados 4 años de fortificación en nuestro medio y 5) describir aparentes modificaciones en el perfil de los defectos congénitos del polo cráneoencefálico durante un periodo de nueve años.

\section{MATERIAL Y MÉTODO}

La Sexta Región de Chile tiene alrededor de 12.000 partos al año y, aproximadamente, la mitad de los nacimientos se verifican en el Hospital Regional de Rancagua. Este último cuenta con registros de diagnóstico prenatal y vigilancia epidemiológica (ECLAMC) desde fines de 1996, lo que permite evaluar en el tiempo la incidencia y características propias de cada uno de los casos que se presentaron en ese periodo.

Para el estudio se consideraron casos todos los recién nacidos $(\mathrm{RN})$ vivos o muertos con más de 500 $\mathrm{g}$ de peso al nacer, con diagnóstico de acranea 0 anencefalia al nacer, de acuerdo al protocolo de registro del ECLAMC. También se consideraron todos aquellos defectos en los cuales existía compromiso encéfalo craneano severo. Estos datos se cruzaron con aquellos que tenían diagnóstico prenatal registrados en la Unidad de Ecografía de nuestro hospital. Se consideraron, además, todos los certificados de defunción de la Dirección Regional de Salud de la VI región, que establecían estos mismos diagnósticos como causa de muerte fetal o neonatal, lo que permite incluir los nacidos de todos los hospitales y clínicas privadas. Sólo los casos que suponían un defecto interno detectado por ecografía prenatal fueron sometidos a una autopsia.

Tanto los fetos como los RN se reclasificaron en 4 subtipos de acuerdo a un patrón fenotípico diferente: A) secuencias acranea/anencefalias clásicas, aisladas, B) acranea/anencefalia asociada a otros DTN, C) defectos cráneo-encefálicos asociados a otras malformaciones mayores y D) defectos cráneoencefálicos asociados a bridas amnióticas (Tabla 1).

De acuerdo a la literatura, la secuencia acranea/ anencefalia sola o asociada con otros DTN, son las más frecuentes y la mayoría pueden estar relacionadas con alteraciones del metabolismo del AF. Los defectos cráneo-encefálicos junto a otros defectos mayores, se asocian a cuadros genético-cromosómicos (síndromes polimalformativos) y los defectos cráneo-encefálicos que presentan lesiones por bridas se relacionan habitualmente con disrupciones por teratógenos (Figuras 1, 2, 3, 4).

Con el total de defectos cráneo-encefálicos reclasificados, se establecieron las tasas anuales por 10.000 nacidos vivos (NV), entre los años 1996 y 2004 de toda la VI región. Para los nacidos durante el año 2000, se consideraron expuestos al ácido fólico, sólo aquellos en que la gestación se inició después de marzo de ese año, como una forma de asegurar que la exposición a la fortificación fue adecuada. Dicho cálculo se realizó comparando retrospectivamente la edad gestacional al nacer con la supuesta fecha de la concepción.

Tabla 1. C lasificación de los defectos de polo cráneo-encefálico

A: acranea con o sin encéfalo (secuencia acranea exencefalia - anencefalia)

B: acranea con o sin encéfalo, asociada a otros DTN (ej: espina bífida, raquisquisis)

C: acranea asociada a otras malformaciones mayores (ej: onfalocele, hernia diafragmática)

D: acranea con encéfalo, asociada a bridas amnióticas (encéfalo placentarias) 


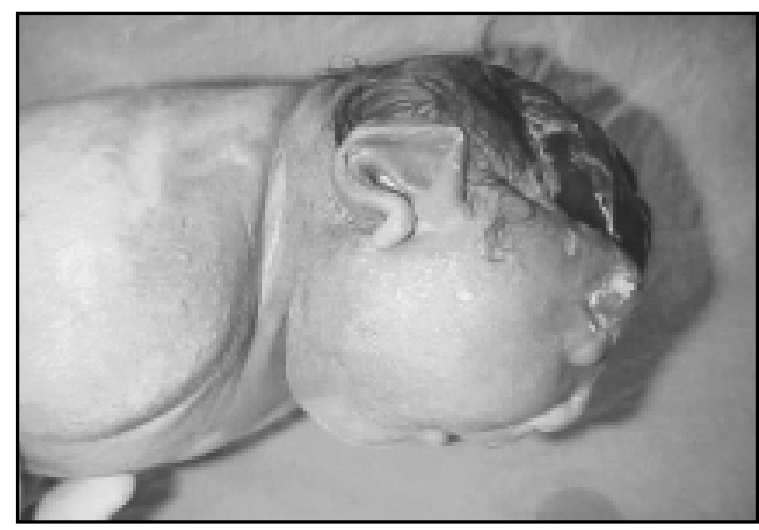

Figura 1. Secuencia acranea/anencefalia (se observa ausencia de encéfalo y calota craneana).

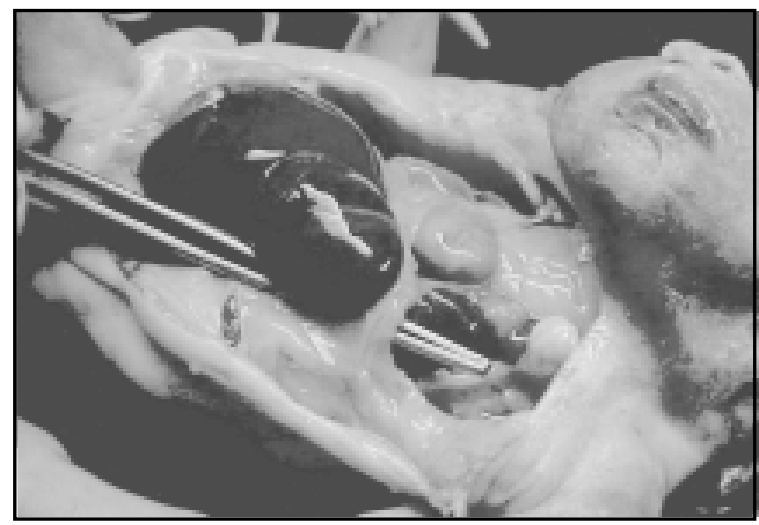

Figura 3. Acranea + otras malformaciones mayores (acranea con encéfalo asociada a hernia diafragmática y cardiopatía congénita).

Se calcularon las tasas anuales y de cada subtipo en ambos periodos y se establecieron los porcentajes de variación entre ellos. Se utilizó programa Epi info versión 3.2.2. para cálculo de riesgo relativo ( $R R$ ) e intervalo de confianza (IC); se aplicó chi cuadrado o test de Fisher y un valor de $\mathrm{p}<0,05$ para establecer el significado estadístico. De esta forma, se evaluó el impacto de la fortificación de la harina, para disminuir el total y cada subtipo de estos defectos congénitos en toda la VI región, permitiendo establecer una medición más segura y objetiva de la frecuencia total y específica de cada defecto.

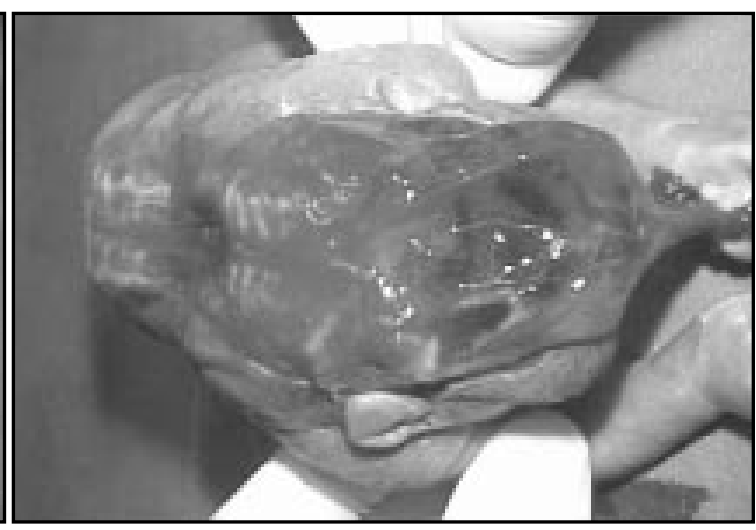

Figura 2. Acranea + DTN (se observa ausencia completa de cráneo y encéfalo con raquisquisis).

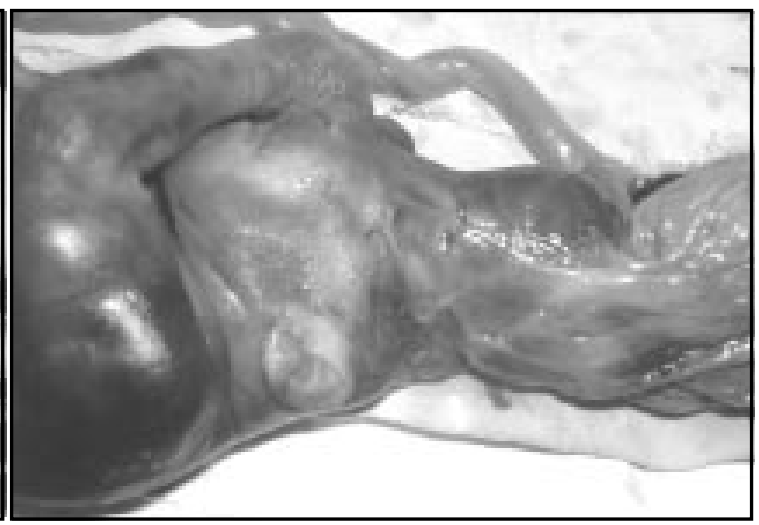

Figura 4. Acranea + brida amniótica (ausencia de cráneo y brida encéfalo placentaria).

\section{Resultados}

Entre 1996 y parte de 2000, considerado el periodo prefortificación, se produjeron 53.056 $\mathrm{RN}$ vivos en toda la VI región y hubo 47 casos de DCCE. Durante el segundo periodo de 2000 y hasta diciembre de 2004, se produjeron 56.120 $\mathrm{RN}$ vivos y ocurrieron 17 casos. En todo el periodo estudiado hubo 109.176 nacimientos y un total de 64 casos de defectos congénitos cráneo-encefálicos. La tasa global fue de 8,86 x $10.000 \mathrm{NV}$ para el periodo prefortificación y de 3,03 x $10.000 \mathrm{NV}$ para el periodo posfor-tificación. 
En la Tabla 2 se observan los registros del total de casos por año y por períodos separados según los diferentes subtipos. Durante el periodo prefortificación 87\% de los casos (41/47) de los grupos A y B, eran defectos cráneo-encefálicos tipo DTN aislados o asociados con otro DTN. El grupo asociado a otras malformaciones (C) y con bridas (D) con 6 casos constuía $13 \%$ del total. Durante el periodo posfortificación, los subtipos A y B representan sólo 47\% (8/ 17) y el grupo C y D 53\% (9/17).

Durante todo el periodo prefortificación se apreciaban discretas variaciones en las tasas anuales, y luego una fuerte caída de la incidencia global en los primeros tres años del periodo posfortificación. El descenso de 47 a 17 casos del primero al segundo periodo establece una disminución global de las tasas de 8,86 x $10.000 \mathrm{NV}$ a $3,03 \times 10.000 \mathrm{NV}$, con una disminución porcentual global de -65,8\%. Esta disminución se hizo fundamentalmente a expensas de los grupos A y B, con una disminución del $-77 \%$ y $-100 \%$, respectiva- mente. La disminución de los subtipos $\mathrm{A}+\mathrm{B}$ juntos fue de $-81 \%$.

Fueron estadísticamente muy significativas la disminución para el grupo total y también la disminución en aquellos subtipos $(\mathrm{A}, \mathrm{B}$ y $\mathrm{A}+\mathrm{B})$ supuestamente los más sensibles al AF. El aumento no significativo en el subtipo D (aquellos defectos asociados a bridas), pudo estar relacionado con el bajo número de la muestra y el uso de teratógenos, y por tanto no modificables con la fortificación de harinas. Por último, el grupo $\mathrm{C}$ registró una discreta disminución porcentual (43\%), sin ser ésta significativa (Tabla 3).

El grupo de defectos no vinculados aparentemente con la fortificación, como los asociados a otras malformaciones (grupo C) se mantuvo prácticamente estable ( 5 y 3 casos, respectivamente). El grupo asociado a bridas (grupo D) aumentó de $2 \%$ a $35 \%$ entre uno y otro periodo (de 1 a 6 casos). Ambos grupos no mostraron variaciones estadísticamente significativas.

Tabla 2. D efectos congénitos cráneo-encefálicos. Subtipos por año y periodo. (Tasas x 10.000 N V)

\begin{tabular}{|lllllllllllll|}
\hline Sub tipo 1996 & 1997 & 1998 & 1999 & 2000 & $\begin{array}{l}\text { Total } \\
\text { pre }\end{array}$ & 2000 & 2001 & 2002 & 2003 & 2004 & $\begin{array}{l}\text { Total } \\
\text { post }\end{array}$ \\
\hline A & 7 & 7 & 9 & 7 & 4 & 34 & 1 & & & 1 & 6 & 8 \\
B & & 2 & 4 & & 1 & 7 & & & & & & 0 \\
C & & 1 & 1 & & 3 & 5 & & 1 & 1 & & 1 & 3 \\
D & & 1 & & & & 1 & 1 & 1 & & 3 & 1 & 6 \\
Total & 7 & 11 & 14 & 7 & 8 & 47 & 2 & 2 & 1 & 4 & 8 & 17 \\
RNV & 13.096 & 12.912 & 12.751 & 12.332 & 1.965 & 53.056 & 10.182 & 11.861 & 11.541 & 11.409 & 11.127 & 56.120 \\
Tasa & 5,34 & 8,51 & 10,97 & 5,67 & & 8,86 & & 1,68 & 0,86 & 3,50 & 7,18 & 3,03 \\
\hline
\end{tabular}

Tabla 3. D efectos cráneo-encefálicos. Tasas y análisis estadístico entre periodo pre y posfortificación. (Tasas x $10.000 \mathrm{~N} \mathrm{~V}$ )

\begin{tabular}{|lccccccl|}
\hline Sub tipo & Tasa pre & Tasa post & \%variación & Valor p-chi & RR & IC 95\% & \\
\hline A & 6,41 & 1,43 & $-77,76$ & 0,000053 & 4,5 & $2,08-9,71$ & S \\
B & 1,32 & 0,00 & $-100,0$ & $0,006^{*}$ & ** & ** & S \\
C & 0,94 & 0,53 & $-43,28$ & $0,333^{*}$ & 1,76 & $0,42-7,38$ & NS \\
D & 0,19 & 1,07 & 467,28 & $0,072^{*}$ & 0,18 & $0,02-1,46$ & NS \\
Total & 8,86 & 3,03 & $-65,80$ & 0,00011 & 2,92 & $1,68-5,09$ & S \\
A+B & 7,73 & 1,43 & $-81,55$ & 0,0000018 & 5,42 & $2,54-11,56$ & S \\
\hline
\end{tabular}

*=Fisher, ${ }^{* *}=$ Indeterminado. 


\section{DisCUSIÓN}

Datos preliminares nacionales, locales y de la Región Metropolitana, establecen disminuciones importantes en la frecuencia al nacer de los DTN luego del periodo de fortificación de la harina con ácido fólico. Sin embargo, estas evaluaciones no separan los defectos en subtipos para ver si corresponden a un DTN clásico, constituyen parte de un síndrome, o están asociadas a bridas encéfalo amnióticas.

Este trabajo reclasifica los defectos congénitos del polo cráneo-encefálico de acuerdo a un patrón fenotípico propio y caracteństico. Se establecen valores sobre la frecuencia de cada subtipo de defecto durante los últimos 9 años y para dos periodos diferentes en la VI región. La disminución de frecuencia es estadísticamente significativa para el total de defectos y mayor aún para los subtipos A, B y A+B. Sin embargo, debemos señalar que existe un ligero aumento de casos del grupo A en el último año, lo que debe onientar hacia la

\section{REFERENCIAS}

1. Compendio Estadístico 2003. Instituto Nacional de Estadísticas. INE Indicadores Bio Demográficos. Pág. 88.

2. Sepúlveda W, Corral E, Ayala C, Be C, Gutiérrez J, VÁsQuez P. Chromosomal abnormalities in fetuses with open neural tube defects: prenatal identification with ultrasound. Ultrasound Obstet Gynecol 2004; 23: 352-6.

3. Cortés F. Prevención primaria de los defectos del tubo neural. Rev Chil Pediatr 2003; 74: 208-12.

4. Nazer J, Aravena T, Cifuentes L Malformaciones congénitas en Chile. Un problema emergente (periodo 1995-1999). Rev Méd Chile 2001; 129: 895-904.

5. Nazer J, Cifuentes L, Meza M. Incidencia de las malformaciones congénitas en 10 maternidades chilenas participantes en el ECLAMC. Comparación de tres periodos (1971-1977, 1982-1988, 1989-1994). Rev Méd Chile 1997; 125: 993-1001.

6. Cortés F, Mellado C, Hertrampf E, Auende A, Castulo S. Frecuencia de los defectos del tubo neural en las maternidades públicas de Santiago durante el año 1999. Rev Méd Chile 2001; 129: 277-84. búsqueda de otros factores, además de los relacionados con la fortificación. Entre éstos se deben revisar variaciones en los hábitos de consumo de haninas de la mujer joven actual, la importación de harinas extranjeras y la participación de teratógenos como la drogadicción en algunos subtipos. Aunque también es posible que estas variaciones se puedan deber a oscilaciones temporales, que se superponen a las medidas de prevención, éstas requieren de estudios con mayor tiempo de observación, ya que, por ejemplo, las tasas de fines del peniodo posfortificación son similares a las del inicio del peniodo prefortificación.

Lo central de este trabajo, primero es la evaluación del impacto que pueda tener una medida preventiva en un espectro malformativo por separado y aun mal definido, como son todos los defectos cráneo-encefálicos. Segundo, la fortificación con ácido fólico tiene un efecto protector importante al disminuir la incidencia de los DCCE tipo DTN clásicos, pero no en todos los defectos cráneo-encefálicos.

7. López-Camelo J, Orioli I, da Graca Dutra M, Nazer J, Rivera N, OJEDA M et al. Reduction of birth prevalence rates of neural tube defects after folic acid fortification in Chile. Am J Med Genet 2005; 135: $120-5$.

8. Freire WB, HertrampF E, Cortes F. Effect of folic acid fortification in Chile: preliminary results. Eur J Pediatr Surg 2000; 10 Suppl 1: 42-3.

9. Moreno R, OJeda ME. Prevención y frecuencia de los defectos del tubo neural. Rev Soc Med VI Región 2003; 2: 482-6.

10. Schneider J, Rees D, Lig Y, Ciegg JB. World wide distribution of a common methylene tetrahydrofolate reductase mutation. Am J Hum Genet 1998; 62: 1258-61.

11. Weitkamp LR, Tackets DC, Hunter AG, Holmes LB, SchwaRTz C. Heterozygote advantage of the MTHFR gene in patients with neural-tube defects and their relatives. Lancet 1998; 351: 1554-5.

12. Nitsche F, Aumende MA, Santos JL, Pérez F, Santa María L, Hertrampf E et al. Frecuencia del polimorfismo C677T de la 5,10-metilen tetrahidrofolato reductasa en mujeres chilenas madres de afectados con espina bífida y en controles normales. Rev Méd Chile 2003; 131: 1399-404. 